\title{
The Role of Glycated Hemoglobin A1c in Determining the Severity of Coronary Artery Disease in Diabetic and Non-Diabetic Subjects in Karachi
}

Ibtehaj Ul-Haque ${ }^{1}$, Zia Ud Deen ${ }^{2}$, Shiza Shafique ${ }^{2}$, Syed Inam Ur Rehman ${ }^{3}$, Maryam Zaman ${ }^{4}$, Syeda T. Basalat ${ }^{2}$, Misbah Munaf ${ }^{2}$, Yusra Wahidi ${ }^{5}$

1. Internal Medicine, Dow University of Health Sciences (DUHS), Karachi, PAK 2. Internal Medicine, Dow University of Health Sciences (DUHS), Karachi, PAK 3. Internal Medicine, Civil Hospital Karachi, Dow University of Health Sciences (DUHS), Karachi, PAK 4. Miscellaneous, Dow University of Health Sciences (DUHS), Karachi, PAK 5. Internal Medicine: Critical Care, Dow University of Health Sciences (DUHS), Karachi, PAK

$\square$ Corresponding author: Ibtehaj Ul-Haque, ibtehaque@gmail.com Disclosures can be found in Additional Information at the end of the article

\section{Abstract \\ Introduction}

Coronary artery disease is the leading cause of death worldwide by incidence. Over the years, many studies have been conducted to find predictors of coronary artery disease; however, in the last few decades, the level of HbA1c in diabetics has been investigated as a potential predictor. Our study offers additional insight by investigating similar relationships in nondiabetic patients and by investigating potential predictors more comprehensively, making it the first of its kind study. The aim of our study is to indicate that rising HbA1c levels suggest that there's a greater risk of coronary artery disease, which can further be confirmed by the SYNTAX score, degree of stenosis, and numbers of vessels involved.

\section{Methods}

Data from 177 diabetic and 378 non-diabetic patients, all of whom were above 18 years of age, were included in the research. HbA1c levels (>5.6\%), SYNTAX score, hypertension, number of vessels involved, and other demographic elements, such as age, smoking, and body measurements, were calculated and compared for diabetics and non-diabetics.

\section{Results}

Received 06/12/2019

Review began 06/17/2019 Review ended 06/19/2019 Published 06/24/2019

\section{(C) Copyright 2019}

Ul-Haque et al. This is an open access article distributed under the terms of the Creative Commons Attribution License CC-BY 3.0., which permits unrestricted use, distribution, and reproduction in any medium, provided the original author and source are credited.
HbA1c was higher in comparison to non-diabetic subjects $(\mathrm{p}<0.001)$. Age $>53$ was found to be a predictor for SYNTAX score $\geqslant 23$ in diabetic patients $(p<0.05)$. Male gender and smoking were found to be independent predictors for three-vessel disease in the non-diabetic population ( $\mathrm{p}$ value $<0.05$ ). There was no significant relationship between the SYNTAX score and HbA1c levels in non-diabetics $(\mathrm{p}=0.885)$ and diabetics. In conclusion, there is no correlation between elevated HbA1c levels and SYNTAX score $\geqslant 23$.

Categories: Cardiology, Endocrinology/Diabetes/Metabolism, Internal Medicine Keywords: diabetes, hba1c, non-diabetics, syntax score, coronary artery disease, cardiology, systolic blood pressure, three-vessel disease, endocrine

\section{How to cite this article}

Ul-Haque I, Ud Deen Z, Shafique S, et al. (June 24, 2019) The Role of Glycated Hemoglobin A1c in Determining the Severity of Coronary Artery Disease in Diabetic and Non-Diabetic Subjects in Karachi. Cureus 11(6): e4982. DOI 10.7759/cureus.4982 


\section{Introduction}

Cardiovascular diseases, specifically coronary artery disease (CAD), over the last 10 years has grown to become the world's largest cause of fatality [1]. The higher incidence and increased risk of developing CAD have been positively linked with diabetes mellitus (DM) in a number of studies [2]. Diabetes mellitus (DM) is a group of metabolic diseases characterized by hyperglycemia resulting from defects in insulin secretion, response to insulin, or both [3]. Glycated haemoglobin (HbA1c) is widely acknowledged as a pivotal and more accurate criterion for pre-empting the severity of DM over fasting glucose levels [4]. HbA1c levels offer more advantage by providing a stable index of long-term glycemic status, which is related to two to three months of average glucose concentration in plasma [5]. Elevated HbA1c levels are notably linked to severe coronary artery atherosclerosis [6-7]. The American Diabetes Association, in its recent position statement, stated that lowering HbA1c may be associated with a reduction in the microvascular, neuropathic, and possibly macrovascular complications of DM [8]. Similarly, since 2004, in non-diabetics, studies linking the relation between the severity of CAD and cardiovascular mortality with HbA1c levels have been widely conducted [5]. Further, they provide evidence that the risk for developing CAD is significantly higher with elevated $\mathrm{HbA1c}$ levels even within the normal range [9].

With consistent evidence that $\mathrm{CAD}$ and HbA1c levels are predictors of cardiovascular mortality, in this study, we used the SYNTAX score and other variables to compare the severity of coronary artery disease in diabetic and non-diabetic patients in a tertiary care hospital in Karachi, Pakistan.

\section{Materials And Methods}

This was a cross-sectional, prospective, and observational study based on a single center. The study was conducted during a time span of 97 days, from January 22, 2018, to April 28, 2018. We randomly selected 177 diabetic patients and 378 non-diabetic patients. All the patients were above the age of 18 years. They had presented to the outpatient department or were admitted to the cardiology ward of Civil Hospital Karachi for clinical reasons, which required them to undergo the coronary angiography procedure. Approval for the protocol of our research was obtained from Pakistan Medical Association Committee (PMA) on Ethics Review Board along with written consent from the cohort involved in our study.

Our exclusion criteria comprised any patient that had already undergone coronary artery bypass, procedures apart from left heart catheterization, and had hemoglobin levels of less than $10.0 \mathrm{gm} \%$.

Hypertension (HTN) and smoking were defined according to the outlines laid by the Joint National Committee in their seventh report on HTN and the interview survey conducted on national health, respectively.

All the patients identified with acute coronary syndrome (ACS) were categorized under unstable angina (USA), non-ST-elevated myocardial infarction (NSTEMI), or ST-elevated myocardial infarction (STEMI), in accordance with the guidelines of the American Heart Association for ACS, 2014. The severity of CAD was graded by cardiologists blinded to the levels of HbA1c [10]. The SYNTAX score was applied for the calculations and anyone with a score of $>22$ was established as a case of severe CAD [11].

We obtained samples of patients for fasting plasma glucose and HbA1c while they were in their overnight fasting state prior to the procedure and classified them into the following divisions based on their HbA1c: Non-Diabetics with HbA1c<5.1, Pre-Diabetics with HbA1c 5.1-6.4, and Diabetics with HbA1c $>6.4$. An exchange chromatography method was used for discerning the 


\section{Cureus}

levels of HbA1c. We considered all the demographic elements such as age, sex, ethnicity, risk factors predisposing to $\mathrm{CAD}$, and varying body measurements. Depending on the patient's choice and accessibility to test at the time, a left ventricular angiogram or echo was used to assess the ejection fraction (EF) of the left ventricle and all angiographic scans were evaluated by well-reputed cardiac specialists.

If any of the major vessels or their branches were found to have more than $50 \%$ stenosis, we designated it as significant CAD and categorized it as follows: Obstructed with $\geqslant 70-100 \%$ stenosis or Non-Obstructed with $>50 \%$ and $<70 \%$ stenosis. Patients were labeled as having either single vessel disease (SVD), double vessel disease (DVD), or triple vessel disease (TVD) based on the number of arteries involved from the three major coronary vessels, namely, Left Anterior Descending, Left Circumflex Artery, and Right Coronary Artery.

The primary incentive was to estimate the relationship of HbA1c values with the severity of CAD.

\section{Results}

In our study, 177 patients had diabetes (D) while 378 patients were non-diabetic (N-D). The majority of the patients, $79.1 \%$ of non-diabetics and $71.2 \%$ of diabetics, were males. The clinical, laboratory, and cardiac investigations of study subjects are mentioned in Table 1 . The results of several clinical and laboratory investigations were found to have strong statistical significance. Diabetic patients had a greater incidence of HTN, with a strong statistically significant difference between the two groups ( $<<0.001, \mathrm{D}=60.4 \%, \mathrm{~N}-\mathrm{D}=43.1 \%$ ). HbA1c levels were higher in diabetic patients with a strong statistical difference between the two groups ( $\mathrm{p}<0.001, \mathrm{D}=8.7 \pm 2.07, \mathrm{~N}-\mathrm{D}=6.0 \pm 1.21$ ). Most diabetic patients had a body mass index (BMI) of more than $25 \mathrm{~kg} / \mathrm{m}^{2}$, the few that were below $25 \mathrm{~kg} / \mathrm{m}^{2}$ revealed statistically significant differences from the non-diabetic group $\left(\mathrm{p}=0.002, \mathrm{D}\left(<25 \mathrm{~kg} / \mathrm{m}^{2}\right)=39.8 \%, \mathrm{~N}-\mathrm{D}\left(<25 \mathrm{~kg} / \mathrm{m}^{2}\right)=\right.$ $50.6 \%)$. Mean waist circumference was slightly greater in diabetic patients with a slight statistically significant difference from the non-diabetic group $(\mathrm{p}=0.002, \mathrm{D}=39.6 \pm 5.03, \mathrm{~N}-$ $\mathrm{D}=38.2 \pm 5.51$ ). Mean systolic blood pressure (SBP) was higher in the diabetic group with a slight statistically significant difference between the groups $(\mathrm{p}=0.023, \mathrm{D}=127 \pm 17.8, \mathrm{~N}-\mathrm{D}=123 \pm 19.8$ ).

\begin{tabular}{|c|c|c|c|c|}
\hline S. No. & & Non-Diabetics (n=378) & Diabetics (n=177) & p-value \\
\hline 1 & Age in years (Mean \pm S.D) & $53.0 \pm 11.21$ & $54.4 \pm 9.38$ & 0.164 \\
\hline \multirow[t]{3}{*}{2} & Gender & & & \\
\hline & Male & $299(79.1 \%)$ & $126(71.2 \%)$ & \multirow{2}{*}{0.052} \\
\hline & Female & $79(20.9 \%)$ & $51(28.8 \%)$ & \\
\hline 3 & HTN & $163(43.1 \%)$ & $107(60.4 \%)$ & 0.001 \\
\hline 4 & Smoker & $170(44.9 \%)$ & $64(36.2 \%)$ & 0.050 \\
\hline 5 & SBP at admission (Mean \pm S.D) & $123 \pm 19.8$ & $127 \pm 17.8$ & 0.023 \\
\hline 6 & DBP at admission (Mean \pm S.D) & $80 \pm 12.6$ & $82 \pm 10.1$ & 0.057 \\
\hline 7 & HbA1c (Mean \pm S.D) & $6.0 \pm 1.21$ & $8.7 \pm 2.07$ & 0.001 \\
\hline 8 & Glucose $(\mathrm{mg} / \mathrm{dl})($ Mean \pm S.D) & $120 \pm 60$ & $114 \pm 23$ & 0.001 \\
\hline
\end{tabular}




\section{Cureus}

BMI

$n=350$

$n=161$

$\leq 25$

$177(50.6 \%)$

$64(39.8 \%)$

0.002

$>25-30$

$117(33.4 \%)$

$67(41.6 \%)$

0.073

$>30$

$56(16.0 \%)$

$30(18.6 \%)$

0.459

Mean \pm S.D

$25.2 \pm 4.98$

$26.7 \pm 5.19$

0.002

Waist Circumference (Mean \pm S.D)

$38.2 \pm 5.51$

$39.6 \pm 5.03$

0.010

Waist /Hip Ratio (Mean \pm S.D)

$0.98 \pm 0.06$

$0.98 \pm 0.05$

0.947

10

SYNTAX Score

$n=160$

$n=79$

$\leq 22$

$119(74.4 \%)$

$50(63.3 \%)$

$\geq 23$

$41(25.6 \%)$

$29(36.7 \%)$

0.007

Mean \pm S.D

$16.1 \pm 10.18$

$20.1 \pm 10.47$

0.005

11

CAD

$n=236$

$n=112$

Normal

$32(13.6 \%)$

$9(8.0 \%)$

0.135

SVD

$80(33.9 \%)$

$21(18.8 \%)$

0.003

DVD

$54(22.9 \%)$

$21(18.8 \%)$

0.381

TVD

$70(29.7 \%)$

$61(54.5 \%)$

0.001

Obstruction

$\mathrm{n}=204$

$n=112$

Non obstructed

$46(22.5 \%)$

$13(12.6 \%)$

Obstructed

$158(77.4 \%)$

$90(87.4 \%)$

12

Types of ACS

$n=370$

$n=168$

USA

$120(33.4 \%)$

$61(36.3 \%)$

NSTEMI

$161(43.5 \%)$

$61(36.3 \%)$

0.469

STEMI

$89(24.1 \%)$

$46(27.4 \%)$

$49.7 \pm 16.51$

$51.5 \pm 15.37$

0.576

\section{TABLE 1: Comparison between diabetics and non-diabetics}

S.D: standard deviation; HTN: hypertension; SBP: systolic blood pressure; DBP: diastolic blood pressure; BMI: body mass index; CAD: coronary artery disease; SVD: single vessel disease; DVD: double vessel disease; TVD: triple vessel disease; ACS: acute coronary syndrome; USA: unstable angina; NSTEMI: non-ST-elevated myocardial infarction; STEMI: ST-elevated myocardial infarction; EF: ejection fraction

Cardiac investigations yielded only a few significant results. The SYNTAX score could only be calculated in 79 diabetic patients and 160 non-diabetic patients. A SYNTAX score of equal to or 


\section{Cureus}

greater than 23 was obtained in $36.7 \%$ and $25.6 \%$ of diabetic and non-diabetic patients with a significant difference between the mean score of two groups $(p=0.005, D=20.1 \pm 10.47, N-D=16.1$ \pm 10.18 ). In $C A D$, the incidence of triple vessel disease was marked in diabetic patients with a strongly significant difference from non-diabetic patients ( $\mathrm{p}<0.001, \mathrm{D}=54.5 \%, \mathrm{~N}-\mathrm{D}=29.7 \%)$ while for single vessel disease, the incidence was greater in non-diabetic patients with a slightly significant difference from the diabetic group ( $p=0.003, D=18.8 \%, N-D=33.9 \%)$. Vessel obstruction was found more commonly in diabetic patients with a slight statistically significant difference from the non-diabetic group $(\mathrm{p}=0.037, \mathrm{D}=87.4 \%, \mathrm{~N}-\mathrm{D}=77.4 \%$ ). The remaining cardiac investigations remained statistically insignificant.

Multivariate logistic regression was calculated for non-diabetic (see Table 2) and diabetic patients (see Table 3) with SYNTAX score $\geqslant 23$ (including male gender, age $\geqslant 53$ years, HTN, smoking, $\mathrm{BMI}>25$, and $\mathrm{HbA} 1 \mathrm{c}>6.4$ as dependent variables). None of the variables were found to be independent predictors for SYNTAX score $\geqslant 23$ in the non-diabetic population while age $>53$ years was an independent predictor for SYNTAX score $\geqslant 23$ in the diabetic population (Beta (age) $=1.432, \mathrm{p}<0.05)$.

\begin{tabular}{|c|c|c|c|c|c|c|}
\hline \multirow{2}{*}{ SYNTAX Score $\geq 23$} & \multirow{2}{*}{ B } & \multirow{2}{*}{ S.E. } & \multirow{2}{*}{ Sig. } & \multirow{2}{*}{$\operatorname{Exp}(B)$} & \multicolumn{2}{|c|}{$95 \%$ C.I for $\operatorname{EXP}(\mathrm{B})$} \\
\hline & & & & & Lower & Upper \\
\hline Male & -.074 & .626 & .905 & .928 & .272 & 3.168 \\
\hline Age $>53$ & .227 & .445 & .610 & 1.254 & .524 & 3.002 \\
\hline Hypertension & -.389 & .474 & .411 & 677 & .268 & 1.714 \\
\hline Smoking & .453 & .503 & .367 & 1.573 & .587 & 4.216 \\
\hline BMI >25 & .044 & .446 & .922 & 1.045 & .436 & 2.505 \\
\hline HbA1c $>6.4$ & .085 & .587 & .885 & 1.088 & .345 & 3.436 \\
\hline 3-Vessel disease & -1.953 & .455 & .000 & .142 & .058 & .346 \\
\hline
\end{tabular}

\section{TABLE 2: Multivariate logistic regression in non-diabetics}

B: beta; S.E: standard error; Sig.: significance; $\operatorname{Exp(B):~exponential~for~beta~coefficient;~C.I:~confidence~interval;~BMI:~body~mass~index~}$ 


\section{Cureus}

\begin{tabular}{|c|c|c|c|c|c|c|}
\hline \multirow{2}{*}{ SYNTAX Score $\geq 23$} & \multirow{2}{*}{$B$} & \multirow{2}{*}{ S.E. } & \multirow{2}{*}{ Sig. } & \multirow{2}{*}{$\operatorname{Exp}(B)$} & \multicolumn{2}{|c|}{$95.0 \%$ C.I. for EXP(B) } \\
\hline & & & & & Lower & Upper \\
\hline Male & -.495 & .737 & .502 & .610 & .144 & 2.583 \\
\hline Age $>53$ & 1.432 & .694 & .039 & 4.189 & 1.074 & 16.333 \\
\hline Hypertension & -.432 & .698 & .536 & .649 & .165 & 2.549 \\
\hline Smoker & -.932 & .668 & .163 & .394 & .106 & 1.458 \\
\hline $\mathrm{BMI}>25$ & .091 & .696 & .896 & 1.096 & .280 & 4.285 \\
\hline $\mathrm{HbA1c}>6.4$ & -.878 & .840 & .296 & .416 & .080 & 2.156 \\
\hline 3-Vessel disease & 2.290 & .841 & .006 & 9.879 & 1.902 & 51.305 \\
\hline
\end{tabular}

\section{TABLE 3: Multivariate logistic regression in diabetics}

B: beta; S.E: standard error; Sig.: significance; $\operatorname{Exp(B):~exponential~for~beta~coefficient;~C.I:~confidence~interval;~BMI:~body~mass~index~}$

Multivariate logistic regression for non-diabetics (see Table 4) and diabetic patients (see Table 5) with three-vessel disease was calculated (including male gender, age $\geqslant 53$, HTN, smoking, BMI $>25$, and $\mathrm{HbA} 1 \mathrm{c}>6.4$ as dependent variables). Male gender and smoking were found to be independent predictors for three-vessel disease in the non-diabetic population (Beta (male) $=$ 1.446 , Beta (smoking) $=-1.268$, p-value $<0.05$ ), whereas no variable was seen to be an independent predictor for 3 -Vessel diseases in diabetic population (Beta $=2.324, \mathrm{p}<0.05)$. 


\section{Cureus}

\begin{tabular}{|c|c|c|c|c|c|c|}
\hline \multirow{2}{*}{ 3-Vessel disease } & \multirow{2}{*}{ B } & \multirow{2}{*}{ S.E. } & \multirow{2}{*}{ Sig. } & \multirow{2}{*}{$\operatorname{Exp}(B)$} & \multicolumn{2}{|c|}{ 95\% C.I. for EXP(B) } \\
\hline & & & & & Lower & Upper \\
\hline Male & 1.446 & .621 & .020 & 4.246 & 1.258 & 14.333 \\
\hline Age $>53$ & -.538 & .434 & .215 & .584 & .250 & 1.367 \\
\hline Hypertension & .230 & .456 & .615 & 1.258 & .514 & 3.079 \\
\hline Smoking & -1.268 & .476 & .008 & .281 & 111 & .715 \\
\hline BMI >25 & -.101 & .444 & .820 & .904 & .379 & 2.157 \\
\hline $\mathrm{HbA1c}>6.4$ & -.913 & .568 & .108 & .401 & 132 & 1.223 \\
\hline SYNTAX Score $\geq 23$ & -1.957 & .456 & .000 & .141 & .058 & .345 \\
\hline
\end{tabular}

\section{TABLE 4: Multivariate logistic regression in non-diabetics}

B: beta; S.E: standard error; Sig.: significance; $\operatorname{Exp(B):~exponential~for~beta~coefficient;~C.I:~confidence~interval;~BMI:~body~mass~index~}$

\begin{tabular}{|c|c|c|c|c|c|c|}
\hline \multirow{2}{*}{ 3-Vessel disease } & \multirow{2}{*}{ B } & \multirow{2}{*}{ S.E. } & \multirow{2}{*}{ Sig. } & \multirow{2}{*}{$\operatorname{Exp}(B)$} & \multicolumn{2}{|c|}{ 95.0\% C.I. for EXP(B) } \\
\hline & & & & & Lower & Upper \\
\hline Male & -.133 & .751 & .860 & .876 & .201 & 3.815 \\
\hline Age $>53$ & .121 & .637 & .850 & 1.128 & .324 & 3.934 \\
\hline Hypertension & .910 & 632 & 150 & 2.485 & .719 & 8.584 \\
\hline Smoker & -.132 & .639 & .836 & .876 & .250 & 3.067 \\
\hline BMI > 25 & .800 & .634 & .207 & 2.225 & .643 & 7.708 \\
\hline HbA1c $>6.4$ & .156 & .787 & .843 & 1.169 & .250 & 5.468 \\
\hline SYNTAX Score $\geq 23$ & 2.324 & .863 & .007 & 10.215 & 1.882 & 55.451 \\
\hline
\end{tabular}

\section{TABLE 5: Multivariate logistic regression in diabetics}

B: beta; S.E: standard error; Sig.: significance; $\operatorname{Exp(B):~exponential~for~beta~coefficient;~C.I:~confidence~interval;~BMI:~body~mass~index~}$

\section{Discussion}

Our study, to the best of our knowledge, is one of the first few studies from Karachi, Pakistan, in which the role of $\mathrm{HbA1c}$ is determined for assessing the severity of CAD in diabetic and non- 
diabetic subjects using the SYNTAX score. In our study, elevated HbA1c did not correlate with the number of vessels involved and the SYNTAX score. The trend showed that, on average, the SYNTAX score was higher in diabetics as compared to non-diabetics. Interestingly, single vessel disease had a greater occurrence in non-diabetics whereas triple vessel disease was more common in diabetics. Subjects were found to have significantly higher HbA1c levels as compared to non-diabetic subjects. Accordingly, the incidence of triple vessel disease and vessel obstructions was significantly higher in diabetic subjects as compared to non-diabetics. A higher incidence of hypertension was also found among diabetic subjects.

Other general clinical and laboratory investigations, such as fasting blood sugar (FBS) and mean systolic blood pressure (SBP), were also measured. Interestingly, FBS levels were lower in diabetic subjects. Mean SBP was also higher in diabetic subjects compared to non-diabetics.

In contrast to our study, Kapil et al. revealed a positive predictor relationship between $\mathrm{HbA} 1 \mathrm{c}$ and cardiovascular disease $(\mathrm{p}=0.001)$ [1]. Moreover, another study from North Eastern India, which used the SYNTAX score also reported a highly significant correlation between elevated HbA1c and higher SYNTAX score [12].

We believe that the significant relationship between male gender and smoking with a threevessel disease in non-diabetic subjects may emphasize a significant mortality rate, which is comparable to several studies that employed HbA1c as a strong indicator of mortality [13] as well as major cardiovascular complications and target vessel revascularization in non-diabetics [14-15]. One of the studies concluded that smoking was a major factor in the mortality of nondiabetic individuals $(p<0.01)[16]$. On the contrary, recent studies have shown that regular advances in cardiovascular medicine, specifically the treatment of acute coronary syndrome (ACS), have contributed to the reduction in morbidity and mortality for acute myocardial infarction (MI) and DM [17].

Most of the studies on this subject employed the Gensini score to determine the severity of CAD while we used the relatively recent and less commonly used scoring modality, the SYNTAX score. The Gensini score determines the degree of stenosis in a vessel as compared to the extent of vessel involvement, which is measured by the SYNTAX score. Although we were not able to produce the desired results from this study, this does not demerit the SYNTAX score; instead, it encourages more medical professionals to conduct further studies to collect more data in order to improve our understanding about the application of this test as outlying results are fairly common for any debatable test.

Similar research that was conducted at the Sanjay Gandhi Postgraduate Institute of Medical Sciences, Lucknow, screened 905 patients with CAD, and their results strongly correlated disease severity and higher SYNTAX score ( $\geqslant 23$ ) with elevated HbA1c levels in diabetics [18]. However, our study was not able to provide a positive correlation between the abovementioned variables. This difference may be due to the limitations of our study, which didn't include the ongoing drug regimen and any previous intervention.

Our research had several limitations. First, our study period was comparatively short. We believe that this provided an inaccurate representation of HbA1c levels, which was further complicated by unavailable information on the duration of diabetes, previous surgeries, and previous/current drug regimen. This might be a major cause as to why HbA1c was a poor predictor in our study. Second, it was not possible to further follow up on our patients; this, too, prevented us from ascertaining the duration of diabetes and other influencing factors.

Reflecting on our shortcomings and based upon the literature search, certain necessary suggestions for fellow researchers are mentioned below: 
- The duration of diabetes should be investigated, as this was found to predict a greater level of coronary vessel occlusions [2].

- Drug regimen and surgical interventions should be included in future studies since these may alter the distribution of coronary lesions, maybe even localizing them. This can potentially decrease the SYNTAX score and provide a false relationship with other variables like HbA1c.

- Additional markers, such as coronary intima-media thickening, glomerular filtration rate (GFR), microalbuminuria, and serum creatinine, should be considered, as they have been successful in pointing toward severe coronary diseases. Carotid intima-media thickness (CIMT) is considered a marker for early atherosclerosis disease, allowing detection in the subclinical stage [1]. Further, microalbuminuria is found to be a strong predictor of the presence and severity of CAD [2].

\section{Conclusions}

The above study suggests that although HbA1c was significantly greater in diabetic patients as compared to non-diabetic patients, this did not positively correlate with the SYNTAX score. This, therefore, demands further investigation into the SYNTAX score as a modality for predicting the severity of coronary artery disease.

\section{Additional Information \\ Disclosures}

Human subjects: Consent was obtained by all participants in this study. Pakistan Medical Association Committee on Ethics issued approval NA. Dated: 7th September' 2018 To, Ibtehajul-Haque, Chief Investigator, Dow Medical College (DMC), Karachi. Subject: Approval for the study on "The role of Glycated Haemoglobin A1c in determining severity of CAD in diabetic and non-diabetic subjects in Karachi" Dear Investigator, With reference to your response dated May 5th, 2018, regarding this proposed observational study. We would like to inform you that PMA Committee on Ethics upon reviewing the study documents noted below, has approved to conduct the study entitled "The role of Glycated Haemoglobin A1c in determining severity of CAD in diabetic and non-diabetic subjects in Karachi”. This cross-sectional study will be conducted at consultant clinics and wards of Civil Hospital, Karachi, Pakistan. You were requested to provide study documents including Study Protocol, Study Questionnaire, Informed Consent Form (ICF) and participating Investigators CVs. The following documents reviewed and approved: • Study Protocol • Informed Consent Form (ICF) • Chief Investigators and Investigators CVs • Study Questionnaire The PMA Committee on Ethics abides by the ICH-GCP guidelines and local regulatory authority. As per the guidelines, the PMA Committee on Ethics Review Board approves this study from an ethical point of view. You must inform PMA Committee on Ethics on a quarterly basis with patient safety reports and any update or amendments to the study documents need to be forwarded to PMA Committee on Ethics. Best Regards Dr. Shershah Syed Chairman PMA Committee on Ethics. Animal subjects: All authors have confirmed that this study did not involve animal subjects or tissue. Conflicts of interest: In compliance with the ICMJE uniform disclosure form, all authors declare the following: Payment/services info: All authors have declared that no financial support was received from any organization for the submitted work. Financial relationships: All authors have declared that they have no financial relationships at present or within the previous three years with any organizations that might have an interest in the submitted work. Other relationships: All authors have declared that there are no other relationships or activities that could appear to have influenced the submitted work.

\section{Acknowledgements}


We would like to thank Dr.Sadaf Ahmed for her continuous support and assistance throughout the research.

\section{References}

1. Kapil C, Rajasekhar D, Vanjakshamma V, Kranthichaitanya D, Narendra CH: Role of CIMT, eGFR, and serum HbA1c in predicting CAD in non-diabetic patients undergoing elective coronary angiography. World J Cardiovasc Dis. 2018, 8: 10.4236/wjcd.2018.81001

2. Mahadeva S, D’Souza SC, Padmanabha K: Comparison of severity of coronary artery disease in diabetic and non-diabetic subjects using Gensini score in Indian subjects. J Diabetes Metab. 2014, 5:469. 10.4172/2155-6156.1000469

3. American Diabetes Association: Diagnosis and classification of diabetes mellitus . Diabetes Care. 2014, 37:81-89. 10.2337/dc10-S062

4. Hong LF, Li XL, Guo YL, et al.: Glycosylated hemoglobin A1c as a marker predicting the severity of coronary artery disease and early outcome in patients with stable angina. Lipids Health Dis. 2014, 13:89. 10.1186/1476-511X-13-89

5. Bastawesy R, Abdelmoniem A, Abdelkader M, Ismaiel R: The relation between glycated hemoglobin and severity of coronary artery disease in non-diabetic patients with acute coronary syndrome. Int J Adv Res. 2016, 4:2393-2399. 10.21474/IJAR01/2663

6. Pu LJ, Lu L, Shen WF, et al.: Increased serum glycated albumin level is associated with the presence and severity of coronary artery disease in type 2 diabetic patients. Circulation J. 2007, 71:1067-1073. 10.1253/circj.71.1067

7. Cai A, Li G, Chen J, Li X, Wei X, Li L, Zhou Y: Glycated hemoglobin level is significantly associated with the severity of coronary artery disease in non-diabetic adults. Lipids Health Dis. 2014, 13:181. 10.1186/1476-511X-13-181

8. American Diabetes Association: Nutrition recommendations and interventions for diabetes. Diabetes Care. 2008, 31:61-78. 10.2337/dc08-S061

9. Verdoia M, Schaffer A, Cassetti E, et al.: Glycosylated hemoglobin and coronary artery disease in patients without diabetes mellitus. Am J Prev Med. 2014, 47:9-16.

10.1016/j.amepre.2014.02.002

10. Amsterdam EA, Wenger NK: The 2014 American College of Cardiology ACC/American Heart Association Guideline for the management of patients with non-ST-elevation acute coronary syndromes. Ten contemporary recommendations to aid clinicians in optimizing patient outcomes. Clin Cardiol. 2015, 38:121-123. 10.1002/clc.22354

11. Kappetein AP, Dawkins KD, Mohr FW, et al.: Current percutaneous coronary intervention and coronary artery bypass grafting practices for three-vessel and left main coronary artery disease. Insights from the SYNTAX run-in phase. Eur J Cardiothorac Surg. 2006, 29:486-491. 10.1016/j.ejcts.2006.01.047

12. Dutta B, Neginhal M, Iqbal F: Glycated hemoglobin (HbA1c) correlation with severity of coronary artery disease in non-diabetic patients-a hospital based study from North-Eastern India. J Clin Diagn Res. 2016, 10:20-23. 10.7860/JCDR/2016/22378.8525

13. Liu Y, Yang YM, Zhu J, Tan HQ, Liang Y, Li JD: Prognostic significance of hemoglobin A1c level in patients hospitalized with coronary artery disease. A systematic review and metaanalysis. Cardiovasc Diabetol. 2011, 10:98. 10.1186/1475-2840-10-98

14. Corpus RA, O'Neill WW, Dixon SR, Timmis GC, Devlin WH: Relation of hemoglobin A1c to rate of major adverse cardiac events in nondiabetic patients undergoing percutaneous coronary revascularization. American J Cardiol. 2003, 92:1282-1286.

10.1016/j.amjcard.2003.08.008

15. Hudson CC, Welsby IJ, Phillips-Bute B, Mathew JP, Lutz A, Hughes GC, Stafford-Smith M: Glycosylated hemoglobin levels and outcome in non-diabetic cardiac surgery patients . Can J Anesth. 2010, 57:565-572. 10.1007/s12630-010-9294-4

16. Chowdhury TA, Lasker SS: Elevated glycated haemoglobin in non-diabetic patients is associated with an increased mortality in myocardial infarction. Postgrad Med J. 1998, 74:480481. 10.1136/pgmj.74.874.480

17. Dubey TN, Mundada K, Arya A: Correlation of HbA1c with mortality and severity in acute coronary syndrome. IJCMR. 2016, 3:2244-2247.

18. Sahal NE, Farrag A, Ammar W, Hegab A: Impact of glycated hemoglobin level on severity of coronary artery disease in non-diabetic patients. J Cardiol Clin Res. 2016, 7:258. 


\section{Cureus}

10.15406/jccr.2016.07.00258 